“ C 2010 IEEE. Personal use of this material is permitted. Permission from IEEE must be obtained for all other uses, in any current or future media, including reprinting/republishing this material for advertising or promotional purposes, creating new collective works, for resale or redistribution to servers or lists, or reuse of any copyrighted component of this work in other works." 


\title{
A Hybrid Adaptive Antenna Array ${ }^{\dagger}$
}

\author{
Xiaojing Huang, Member, IEEE, Y. Jay Guo, Senior Member, IEEE, \\ and John Bunton, Senior Member, IEEE \\ CSIRO ICT Centre
}

Australia

Abstract - Owing to the excessive demand on signal processing and space constraint, a full digital implementation of a large adaptive antenna array is very challenging, especially at millimetre wave frequencies. To overcome the difficulty, a novel hybrid adaptive antenna array is presented, which consists of analogue subarrays followed by a digital beamformer. Two subarray configurations, the interleaved subarray and the side-by-side subarray, are proposed, and the corresponding adaptive angle of arrival estimation and beamforming algorithms, the differential beam tracking and the differential beam search, are developed. The performance of the algorithms is evaluated using a proposed recursive mean square error bound and demonstrated by simulation.

Index Terms—Adaptive antenna array, subarray, beamforming, angle of arrival estimation, and mmwave communications.

\section{INTRODUCTION}

With the growing demand for long range and high data rate wireless communications and the advance in digital signal processing techniques, adaptive antenna arrays have found a wide rage of applications and are becoming an essential part of the wireless communications systems [1-3]. The use of adaptive antenna arrays for millimetre wave (mm-wave) mobile and ad hoc communications networks, such as those providing wireless connectivity between aircrafts and/or between aircraft and ground vehicle or control station, is particularly critical due to the limited output power of the monolithic microwave integrated circuits (MMIC) [4,5]. Combing multiple antennas, each of which has its own low noise amplifier (LNAs) or power amplifiers (PAs) to form an antenna array, not only increases the communications range but also enables the digital beamforming technology to be applied to optimize the system performance.

\footnotetext{
The research is partially funded by Boeing Company.
} 
Since the antenna elements in an array must be placed closely together to prevent grating lobes, the analogue components, such as the LNAs, PAs and the down (or up) converters associated with each antenna element, must be tightly packed behind the antenna elements. This space constraint appears to be a major engineering challenge at mm-wave frequencies. For example, at 55 and $95 \mathrm{GHz}$, the required element spacing is only 2.9 and $1.7 \mathrm{~mm}$, respectively. With the current MMIC technology, the practical implementation of such a digital antenna array remains very difficult $[6,7]$. Another issue with pure digital beamformers is the excessive demand on real time signal processing for high gain antennas. To achieve an antenna gain of over $30 \mathrm{~dB}$, for instance, one may need more than 1000 antenna elements. This makes most beanforming algorithms impractical for commercial applications. Furthermore, to perform wideband digital beamforming, each signal from/to an antenna element is normally divided into a number of narrowband signals and processed separately, which also adds to the cost of digital signal processing significantly. Therefore, a full digital implementation of large, wideband antenna array at mm-wave frequencies is simply unrealistic [8].

In this paper, a novel hybrid adaptive receive antenna array is proposed to solve the digital implementation complexity problem in large arrays. In the hybrid antenna array, a large number of antenna elements are grouped into analogue subarrays. Each subarray uses an analogue beamformer to produce beamformed subarry signal, and all subarray signals are combined using a digital beamformer to produce the final beamformed signal [9]. Each element in a subarry has its own radio frequency (RF) chain and employs an analogue phase shifting device at the intermediate frequency (IF) stage. Signals received by all elements in a subarry are combined after analogue phase shifting, and the analogue beamformed signal is down-converted to baseband and then converted into digital domain. In this way, the complexity of the digital beamformer is reduced by a factor equal to the number of elements in a subaray. For example, for a 1024 element hybrid array of 64 subarrays each having 16 elements, only 64 inputs to the digital beamformer are necessary, and the complexity is reduced to one sixteenth for algorithms of linear complexity, such as the least mean squire (LMS) algorithm, as well as digital hardware cost. The digital beamformer estimates the angle of arrival (AoA) information to control the phases of the 
phase shifters in the analogue subarrays and also adjusts the digital weights applied to the subarray output signals to form a beam. It should be noted that the subarray technology has been used over the past decades [2, 10-12]. Major ideas include employing a time delay unit to each phased subarray for bandwidth enhancement, and eliminating phase shifters in the subarray for applications requiring only limited-field-of-view. The proposed hybrid antenna array concept differs in that it is a new architecture allowing the analogue subarrays and the low complexity digital beamformer to interact with each other to accommodate the current digital signal processing capability and MMIC technology, thus enabling the implementation of a large adaptive antenna array.

The AoA estimation and beamforming algorithms suitable for the proposed hybrid antenna architecture are also significantly different from the conventional ones. First, since the inputs to the digital beamformer are the analogue beamformed signals which are obtained based on a previously estimated AoA, the AoA estimation in the digital beamformer must be recursive in nature. Second, since only the line-of-sight (LOS) incident beam needs to be considered in a point-to-point link which is the targeted application of this hybrid array, the AoA estimation can be performed more efficiently than the conventional AoA estimation techniques [13-18] which can be classified into two categories, i.e., the spatial spectral based and the parametric approaches. The spatial spectral based algorithms, such as the Bartlett beamforming [13] and the various subspace-based methods (e.g., MUSIC $[15,16]$ ), need to obtain a spectrum of the AoAs and then find the spectral peaks, whereas the parametric methods, e.g., the ones based on maximum likelihood (ML) principle [17,18], require multi-dimensional search to solve an optimization problem in which the global convergence may not be guaranteed. These conventional techniques are too costly to use in a complexity-reduced digital implementation of a large array.

Two types of hybrid antenna arrays with different subarray configurations are proposed in the paper. One is the hybrid array of interleaved subarrays and the other is the hybrid array of side-by-side subarrays. Two adaptive AoA estimation algorithms, referred to as differential beam tracking (DBT) and differential beam search (DBS), are developed accordingly. The algorithms make use of the phase difference between the adjacent received subarray outputs to estimate the 
AoA information, which removes the necessity of a known reference signal or signal synchronization at low signal-to-noise ratios (SNR) during the initial beam acquisition, whereas for conventional adaptive beamforming techniques, such as LMS and iterative beam steering (IBS) algorithms [1,2], a known reference signal and signal synchronization are necessary. The DBT algorithm can be applied for both beam acquisition and tracking when interleaved subarrays are employed. The DBS algorithm incorporates an effective beam search strategy to solve the phase ambiguity problem which is inherent to the more practical side-by-side subarray configuration. It is used for beam acquisition first, and then for beam tracking. To analyse the performance of the proposed algorithms, a recursive mean square error bound (MSEB) is derived, which solves the difficult problem of evaluating the mean square error (MSE) of the phase estimation in the presence of recursive nuisance parameters.

The rest of the paper is organized as follows. In Section II, two different configurations of the hybrid adaptive array are described and the received signal models for subarray output signals and overall array output signal are given. Section III presents the principle of the proposed adaptive AoA estimation and gives the detailed beam tracking and search algorithms. Section IV formulates the AoA estimation as a phase estimation problem in the presence of recursive nuisance parameters and derives the recursive MSEB for the estimation. Section V provides the simulation results to demonstrate the performance of the proposed algorithms. Finally, conclusions are drawn in Section V.

\section{HyBrid ARRAY CONFIGURATIONS AND SignAL MODELS}

\section{A. Hybrid Array of Subarrays}

A subarray is a subset of elements in an antenna array [2]. In the presented research, each element in a subarray is connected to an analogue phase shifter. The received signals from individual elements after phase shifting are combined to produce the output signal of the subarray, which is called the analogue beamforming. The proposed hybrid array can be constructed by two or more such subarrays. The subarrays can be interleaved or placed side-by-side with each other. Some examples of the hybrid arrays of subarrays are illustrated in Fig. 1, where the distance 
between adjacent elements in a subarray is referred to as the subarray element spacing and the distance between corresponding elements in adjacent subarrays is termed the subarray spacing. For the hybrid arrays of interleaved subarrays shown in Fig. 1 the subarray element spacing is $2 d$ and the subarray spacing is $d$, whereas for the hybrid arrays of side-by-side subarrays shown in Fig. 1 the subarray element spacing is $d$ and the subarray spacing is $4 d$.

The subarray output signals are converted into digital signals at baseband via analogue-todigital converters (A/D). Then, digital beamforming is performed to control the phase shifters in the subarrays as well as the digital weights associated with respective subarray output signals. The hybrid beamformer structure is illustrated in Fig. 2 using the linear array of two side-by-side subarrays, where RF and down-conversion devices are not shown for simplicity.

\section{B. Received Signal Models}

Denoting the received signal of the $m$ th subarray, $m=0,1, \cdots, M-1$, where $M$ is the total number of subarrays, as $s_{m}(t)$, and the received information-bearing signal as $\tilde{s}(t)$, the received subarray signal can be expressed as

$$
S_{m}(t)=\tilde{s}(t) \sum_{i=0}^{N-1} P_{i, m}(\theta, \phi) e^{j\left[\frac{2 \pi}{\lambda_{c}}\left(X_{i, m} \sin \theta \cos \phi+Y_{i, m} \sin \theta \sin \phi\right)+\alpha_{i, m}\right]}+n_{m}(t), \quad m=0,1, \cdots, M-1,
$$

where $N$ is the total number of elements in a subarray, $P_{i, m}(\theta, \phi)$ is the radiation pattern of the $i$ th element located at $\left(X_{i, m}, Y_{i, m}\right)$ in the $m$ th subarray, $\theta$ and $\phi$ are the zenith and azimuth angles respectively, $\lambda_{c}$ is the wavelength of the RF signal, $\alpha_{i, m}$ is the phase shifted by the $i$ th phase shifter of the $m$ th subarray, and $n_{m}(t)$ is the total additive white Gaussian noise presented at the output of the $m$ th subarray.

Furthermore, ignoring the mutual coupling between elements and other antenna imperfection, we assume that all subarrays are the same, i.e., $P_{i, m}(\theta, \phi)=P_{i}(\theta, \phi)$ and $\alpha_{i, m}=\alpha_{i}$. Also, we assume that the number of subarrays can be expressed as $M=M_{x} \times M_{y}$, where $M_{x}$ and $M_{y}$ are the numbers of subarrays placed along x-axis and y-axis respectively, and that the locations of the $i$ th elements in the $m$ th subarray, $m=m_{y} M_{x}+m_{x}$, are arranged such that 
$X_{i, m}=X_{i}+m_{x} d_{x}^{s}, m_{x}=0,1, \cdots, M_{x}-1$, and $Y_{i, m}=Y_{i}+m_{y} d_{y}^{s}, m_{y}=0,1, \cdots, M_{y}-1$, where $d_{x}^{s}$

and $d_{y}^{s}$ are the subarray spacings along $\mathrm{x}$-axis and $\mathrm{y}$-axis respectively, and $\left(X_{i}, Y_{i}\right)$ is the location of the $i$ th element of the subarray numbered $m=0$. Then, (1) can be simplified as

$$
S_{m}(t)=\tilde{s}(t) P_{s}(\theta, \phi) e^{j \frac{2 \pi}{\lambda_{c}}\left(m_{x} d_{x}^{s} \sin \theta \cos \phi+m_{y} d_{y}^{s} \sin \theta \sin \phi\right)}+n_{m}(t)
$$

where

$$
P_{s}(\theta, \phi)=\sum_{i=0}^{N-1} P_{i}(\theta, \phi) e^{j\left[\frac{2 \pi}{\lambda_{c}}\left(X_{i} \sin \theta \cos \phi+Y_{i} \sin \theta \sin \phi\right)+\alpha_{i}\right]}
$$

is the overall radiation pattern of a subarray.

In general, a two-dimensional subarray can have $N=N_{x} \times N_{y}$ elements, where $N_{x}$ and $N_{y}$ are the numbers of elements placed along $\mathrm{x}$-axis and $\mathrm{y}$-axis respectively. The location $\left(X_{i}, Y_{i}\right)$ of the $i$ th element, $i=i_{y} N_{x}+i_{x}$, is given by $X_{i}=X_{0}+i_{x} d_{x}^{e}, i_{x}=0,1, \cdots, N_{x}-1$, and $Y_{i}=Y_{0}+i_{y} d_{y}^{e}, i_{y}=0,1, \cdots, N_{y}-1$, where $d_{x}^{e}$ and $d_{y}^{e}$ are the subarray element spacings along Xaxis and y-axis respectively, and $\left(X_{0}, Y_{0}\right)$ is the location of the element numbered $i=0$.

Finally, denoting the digital weight applied to the $m$ th subarray signal as $w_{m}^{*}$, the overall digital beam formed output signal at $t=n T$, where $T$ is the sampling period, is

$$
s[n]=\sum_{m=0}^{M-1} w_{m}^{*} s_{m}(n T)=\sum_{m=0}^{M-1} w_{m}^{*} s_{m}[n]
$$

where $s_{m}[n]=s_{m}(n T)$ is the sampled subarray signal in the digital domain.

\section{Subarray Radiation Pattern}

Without loss of generality, we assume that the antenna array consists of isotropic elements with omni-directional radiation patterns, i.e., $P_{i}(\theta, \phi)=1$. Then, the radiation pattern expressed in (3) is the same as the array factor of the subarray. When the phase shifts of the elements in a subarray are chosen as $\alpha_{i}=-\frac{2 \pi}{\lambda_{c}}\left(X_{i} \sin \theta_{0} \cos \phi_{0}+Y_{i} \sin \theta_{0} \sin \phi_{0}\right)$, which allows the main beam of the array to be directed towards the direction represented by the angles $\left(\theta_{0}, \phi_{0}\right)$, and $\left(X_{0}, Y_{0}\right)$ is 
chosen so that the subarray is centred about the origin of the $x-y$ coordinates, the normalized radiation pattern of a subarray can be expressed as the well known form

$$
\begin{aligned}
\bar{P}_{s}(\theta, \phi)=\frac{P_{s}(\theta, \phi)}{N_{x} N_{y}}= & \frac{\sin \left(N_{x} \frac{\pi}{\lambda_{c}} d_{x}^{e}\left(\sin \theta \cos \phi-\sin \theta_{0} \cos \phi_{0}\right)\right)}{N_{x} \sin \left(\frac{\pi}{\lambda_{c}} d_{x}^{e}\left(\sin \theta \cos \phi-\sin \theta_{0} \cos \phi_{0}\right)\right)} \\
& \cdot \frac{\sin \left(N_{y} \frac{\pi}{\lambda_{c}} d_{y}^{e}\left(\sin \theta \sin \phi-\sin \theta_{0} \sin \phi_{0}\right)\right)}{N_{y} \sin \left(\frac{\pi}{\lambda_{c}} d_{y}^{e}\left(\sin \theta \sin \phi-\sin \theta_{0} \sin \phi_{0}\right)\right)} .
\end{aligned}
$$

For a two-dimensional hybrid array of interleaved subarrays, we have $d_{x}^{e}=M_{x} d$ and $d_{y}^{e}=M_{y} d$, where $d$ is the element spacing of the hybrid array, which is also the same as the subarray spacing along $\mathrm{x}$-axis or $\mathrm{y}$-axis, i.e., $d_{x}^{s}=d_{y}^{s}=d$. For a two-dimensional hybrid array of side-by-side subarrays, we have $d_{x}^{e}=d_{x}^{e}=d$, whereas the subarray spacings along x-axis and yaxis are $d_{x}^{s}=N_{x} d$ and $d_{y}^{s}=N_{y} d$, respectively.

\section{AOA ESTIMATION AND BEAMFORMING}

\section{A. Differential Beam Tracking (DBT)}

Let's first consider the hybrid array of interleaved subarrays. From the subarray output signal model (2), it is observed that the inputs to the digital beamformer are affected by the subarray radiation pattern which is determined by the AoA $(\theta, \phi)$ as well as an initial AoA estimate $\left(\theta_{0}, \phi_{0}\right)$. Therefore, any further AoA estimation can only be made based on a previously estimated AoA, which means that the AoA estimation for the hybrid array must be recursive in nature. It is also observed that, at a given initial AoA, the AoA information can be estimated from the phase difference between the adjacent subarray output signals provided that $P_{s}(\theta, \phi) \neq 0$. Thus, by taking the cross-correlation of any two adjacent subarray output signals along $\mathrm{x}$-axis and $\mathrm{y}$-axis respectively and assuming that the noise components are independent, we have 


$$
R_{x}=E\left\{S_{m_{y} M_{x}+m_{x}}^{*}(t) S_{m_{y} M_{x}+m_{x}+1}(t)\right\}=\left.E\left\{\left.\tilde{s}(t)\right|^{2}\right\} P_{s}(\theta, \phi)\right|^{2} e^{j \frac{2 \pi}{\lambda_{c}} d_{x}^{s} \sin \theta \cos \phi}
$$

and

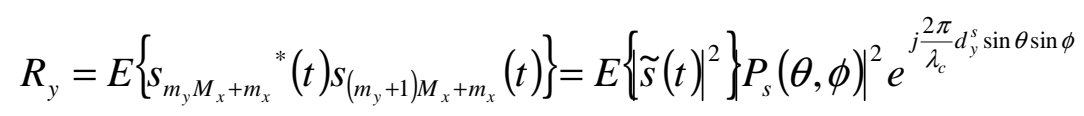

where $E\{\{\}$ denotes ensemble expectation.

Since $d_{x}^{s}=d_{y}^{s}=d$ and assuming $d \leq \frac{\lambda_{c}}{2}$, the following variables

$$
\begin{aligned}
& u_{x}=\frac{2 \pi}{\lambda_{c}} d \sin \theta \cos \phi \\
& u_{y}=\frac{2 \pi}{\lambda_{c}} d \sin \theta \sin \phi
\end{aligned}
$$

contain the AoA information of the incident signal and will take on values in the range $[-\pi, \pi)$. They can be obtained from $\arg \left\{R_{x}\right\}$ and $\arg \left\{R_{y}\right\}$ respectively without any ambiguity, i.e.,

$$
\begin{aligned}
& u_{x}=\arg \left\{R_{x}\right\} \\
& u_{y}=\arg \left\{R_{y}\right\} .
\end{aligned}
$$

The obtained $u_{x}$ and $u_{y}$ can be used to determine the phase shifts in the subarrays. The explicit values of the angles $-\frac{\pi}{2} \leq \theta \leq \frac{\pi}{2}$ and $-\frac{\pi}{2} \leq \phi \leq \frac{\pi}{2}$ are not required though they can be easily determined from $u_{x}$ and $u_{y}$ as $\theta=\operatorname{sign}\left(u_{x}\right) \sin ^{-1}\left(\frac{\lambda_{c}}{2 \pi} \frac{\sqrt{u_{x}^{2}+u_{y}^{2}}}{d}\right)$ and $\phi=\operatorname{tg}^{-1}\left(\frac{u_{y}}{u_{x}}\right)$.

In the digital domain, the cross-correlations along $\mathrm{x}$-axis and $\mathrm{y}$-axis can be estimated iteratively using the digital subarray output signals sampled at $t=n T$ as

$$
\begin{aligned}
& R_{x}^{(n)}=(1-\mu) R_{x}^{(n-1)}+\mu \sum_{m_{y}=0}^{M_{y}-1} \sum_{m_{x}=0}^{M_{x}-2} s_{m_{y} M_{x}+m_{x}}{ }^{*}[n] s_{m_{y} M_{x}+m_{x}+1}[n] \\
& R_{y}^{(n)}=(1-\mu) R_{y}^{(n-1)}+\mu \sum_{m_{x}=0}^{M_{x}-1} \sum_{m_{y}=0}^{M_{y}-2} s_{m_{y} M_{x}+m_{x}}{ }^{*}[n] S_{\left(m_{y}+1\right) M_{x}+m_{x}}[n]
\end{aligned}
$$


where $0<\mu<1$ is the updating coefficient. All available subarray outputs are used for the crosscorrelation estimation in order to improve the SNR.

After applying the digital weights $w_{m}^{*}=e^{-j\left(m_{x} u_{x}+m_{y} u_{y}\right)}, m=m_{y} M_{x}+m_{x}$, to the subarray output signals, the beamformed signal can be obtained by (4) accordingly.

The algorithm for analogue phase shifter control is now summarized as follows.

1. Update $R_{x}^{(n)}$ and $R_{y}^{(n)}$ using (12) and (13);

2. Calculate $u_{x}^{(n)}=\arg \left\{R_{x}^{(n)}\right\}$ and $u_{y}^{(n)}=\arg \left\{R_{y}^{(n)}\right\}$;

3. Determine the subarray phase shifts $\alpha_{i}^{(n)}=-\frac{X_{i} u_{x}^{(n)}+Y_{i} u_{y}^{(n)}}{d}$.

Since the above algorithm uses the phase difference between adjacent subarray output signals to obtain the AoA information and to track the AoA adaptively, we call it differential beam tracking. It is a blind algorithm since no knowledge about the reference signal $\tilde{s}(t)$ is required.

\section{B. Phase Ambiguity and Beam Scanning}

For the hybrid antenna array of side-by-side subarrays, the subarray spacings are $d_{x}^{s}=N_{x} d$ and $d_{y}^{s}=N_{y} d$. Therefore, the phases of $R_{x}$ and/or $R_{y}$ can be outside the range $[-\pi, \pi)$, and ambiguity will occur when $\arg \left\{R_{x}\right\}$ and $\arg \left\{R_{y}\right\}$ are used to determine the phases of $R_{x}$ and $R_{y}$ respectively.

To remove this ambiguity and thus obtain the correct AoA information, one can find all the possible $u_{x}$ and $u_{y}$ values from $\arg \left\{R_{x}\right\}$ and $\arg \left\{R_{y}\right\}$ respectively, and try all the possible combinations of $u_{x}$ and $u_{y}$, which represent all the possible beams, to see which combination gives the beam with the maximum output power. The $u_{x}$ and $u_{y}$ values corresponding to the largest beamformed signal power is used to obtain the AoA information.

All the possible $u_{x}$ and $u_{y}$ values can be determined respectively by

$$
u_{x}(p)=\frac{2 \pi p+\arg \left\{R_{x}\right\}}{N_{x}}, \quad p=-\left[\frac{N_{x}}{2}\right],-\left[\frac{N_{x}}{2}\right]+1, \cdots, 0,1, \cdots\left[\frac{N_{x}}{2}\right]
$$




$$
u_{y}(q)=\frac{2 \pi q+\arg \left\{R_{y}\right\}}{N_{y}}, \quad q=-\left[\frac{N_{y}}{2}\right],-\left[\frac{N_{y}}{2}\right]+1, \cdots, 0,1, \cdots\left[\frac{N_{y}}{2}\right]
$$

where [.] denotes the operation of taking the integer part of a value. Each combination of $u_{x}(p)$ and $u_{y}(q)$ represents a possible beam. The phase shifts for given $p$ and $q$

$$
\alpha_{i}(p, q)=-\frac{X_{i} u_{x}(p)+Y_{i} u_{y}(q)}{d}
$$

are applied to control the subarrays towards the selected beam, and the beam formed signal at $t=n T$ can be obtained accordingly as

$$
s[n, p, q]=\sum_{m_{y}=0}^{M_{y}-1} \sum_{m_{x}=0}^{M_{x}-1} e^{-j\left(m_{x} N_{x} u_{x}^{(n)}(p)+m_{y} N_{y} u_{y}^{(n)}(q)\right)} S_{m_{y} M_{x}+m_{x}}[n]
$$

where $u_{x}^{(n)}(p)$ and $u_{y}^{(n)}(q)$ denote $u_{x}(p)$ and $u_{y}(q)$ obtained at $t=n T$, respectively.

To compare the signal powers of different beams, all possible beams are scanned within a period of time. We call this period of time the scanning frame. A scanning frame is divided into subframes for different combinations of $p$ and $q$. Within each subframe, each beam formed signal power is calculated. At the end of a scanning frame, the beam with the largest signal power is decided as the estimated signal beam. The beam scanning frame and subframe are illustrated in Fig. 3 for a linear subarray with 5 elements $\left(N=N_{x}=5\right.$ and $\left.N_{y}=1\right)$ and $\arg \left\{R_{x}\right\}=\frac{\pi}{3}$. In this example, there are 5 possible beams with $u_{x}(-2)=-\frac{11 \pi}{15}, u_{x}(-1)=-\frac{\pi}{3}, u_{x}(0)=\frac{\pi}{15}, u_{x}(1)=\frac{7 \pi}{15}$, and $u_{x}(2)=\frac{13 \pi}{15}$ according to (14). The scanning frame has 5 subframes and the beams are scanned from $u_{x}(-2)$ to $u_{x}(2)$ in order.

\section{Differential Beam Search (DBS)}

As in the DBT algorithm, the cross-correlations $R_{x}$ and $R_{y}$ can be still iteratively estimated using (12) and (13) in the digital domain, whereas the beamformed signal power $\sigma_{s}^{2}(p, q)=E\left\{s[n, p, q]^{2}\right\}$ can be iteratively estimated in a subframe by 


$$
\sigma_{s}^{2(n)}(p, q)=(1-\beta) \sigma_{s}^{2(n-1)}(p, q)+\beta \mid s[n, p, q]^{2}
$$

where $0<\beta<1$ is the updating coefficient.

Combining the above iterations (12), (13), (17) and (18) with the beam scanning scheme, one obtains the adaptive algorithm, called the differential beam search, which proceeds in a subframe for a given combination of $p$ and $q$ as follows at the $n$th iteration.

1. Update $R_{x}^{(n)}$ and $R_{y}^{(n)}$ using (12) and (13);

2. Calculate $u_{x}^{(n)}(p)=\frac{2 \pi p+\arg \left\{R_{x}^{(n)}\right\}}{N_{x}}$ and $u_{y}^{(n)}(q)=\frac{2 \pi q+\arg \left\{R_{y}^{(n)}\right\}}{N_{y}}$;

3. Determine $\alpha_{i}^{(n)}(p, q)=-\frac{X_{i} u_{x}^{(n)}(p)+Y_{i} u_{y}^{(n)}(q)}{d}$;

4. Update $s[n, p, q]$ and $\sigma_{s}^{2(n)}(p, q)$ using (17) and (18);

5. Select $p$ and $q$ for next subframe.

The DBS algorithm is also a blind algorithm since no knowledge of the reference signal is assumed. It can be seen as a generalised DBT in which the beam is locked to the only possible one without ambiguity.

If the length of a subframe is chosen so that the power of each beam can be calculate with sufficient accuracy, one scanning frame will be sufficient to determine the most likely beam. If shorter subframes are used, the scanning frame can be repeated until the power of each beam calculated across multiple scanning frames is obtained with sufficient accuracy. Once the correct beam is determined, the DBT algorithm can then be used to track the change of the selected beam. Due to the delay from the time when the phase shifts are loaded into the phase shifters in subarrays to the time when a change of the beamformed signal is observed, a minimum length of the subframe will be required.

\section{Performance Evaluation}

\section{A. Formulation of Blind AoA Estimation Problem}


The performance of the proposed blind AoA estimation algorithms can be measured by the MSE of the estimated $u_{x}$ (or $u_{y}$ ). To simplify the analysis, we only consider a linear hybrid array of two interleaved subarrays with $N=N_{x}$ elements. We also assume that the incident beam is pointed at $\theta=0$ and the AoA remains unchanged during the duration of estimation. Therefore, the normalized subarray radiation pattern is only a function of $u=u_{x}=\frac{2 \pi}{\lambda_{c}} d \sin \theta_{0}$ and is thus denoted as $\bar{P}_{s}(u)=\frac{\sin (N u)}{N \sin (u)}$. Under the above assumption, the received subarray signals in the digital domain at time index $n$ can be expressed as

$$
\begin{aligned}
& s_{0}[n]=\tilde{s}[n] \bar{P}_{s}\left(u^{(n-1)}\right)+z_{0}[n] \\
& s_{1}[n]=\tilde{s}[n] \bar{P}_{s}\left(u^{(n-1)}\right) e^{j u}+z_{1}[n]
\end{aligned}
$$

where $\tilde{s}[n]=\tilde{s}(n T)$ is the reference signal sampled at $t=n T$ with average power $\sigma_{\tilde{s}}^{2}=E\left\{\left[\tilde{s}[n]^{2}\right\}\right.$, $u^{(n-1)}$ denotes the variable $u$ estimated at time index $n-1, z_{0}[n]$ and $z_{1}[n]$ are independent complex Gaussian noises with zero mean and the same noise power $\sigma_{z}^{2}$. Note that, compared with (2), the normalized subarray radiation pattern $\bar{P}_{s}\left(u^{(n-1)}\right)$ is used in the above signal models since the scaling factor can be absorbed into $\tilde{s}[n]$ and thus the SNR in a subarray can be expressed as $\gamma=\frac{\sigma_{\tilde{s}}^{2}}{\sigma_{z}^{2}}$ after the subarray is correctly beamformed.

The differential signal between $s_{0}[n]$ and $s_{1}[n]$ is

$$
\begin{aligned}
r(n) & =s_{0}{ }^{*}[n] S_{1}[n] \\
& =\left.\left|\tilde{s}[n]^{2}\right| \bar{P}_{s}\left(u^{(n-1)}\right)\right|^{2} e^{j u}+\tilde{s}[n] \bar{P}_{s}\left(u^{(n-1)}\right) e^{j u} z_{0}{ }^{*}[n]+\tilde{s}^{*}[n] \bar{P}_{s}^{*}\left(u^{(n-1)}\right) z_{1}[n]+z_{0}{ }^{*}[n] z_{1}[n] \\
& =|\tilde{s}[n]|^{2}\left|\overline{P_{s}}\left(u^{(n-1)}\right)\right|^{2} e^{j u}+z[n]
\end{aligned}
$$

where

$$
z[n]=\tilde{s}[n] \bar{P}_{s}\left(u^{(n-1)}\right) e^{j u} z_{0}^{*}[n]+\tilde{s}^{*}[n] \bar{P}_{s}^{*}\left(u^{(n-1)}\right) z_{1}[n]+z_{0}{ }^{*}[n] z_{1}[n]
$$


can be approximated as a complex Gaussian noise with zero mean and total noise power $2|\tilde{s}[n]|^{2}\left|\bar{P}_{s}\left(u^{(n-1)}\right)\right|^{2} \sigma_{z}^{2}$.

From (21), the estimation of $u$ is formulated as the phase estimation of $r(n)$ based on the observed $r(k), k=1,2, \cdots, n$, in the presence of random nuisance parameters $\tilde{s}(k)$ and previously estimated $u^{(k-1)}, \quad k=1,2, \cdots, n$.

\section{B. Recursive MSE Bound}

According to the DBT algorithm based on the obtained differential data set $r(k)$, $k=1,2, \cdots, n$, the non-coherent estimation of $u$ is given by

$$
u^{(n)}=\arg \left\{R^{(n)}\right\}
$$

where

$$
R^{(n)}=\sum_{k=1}^{n} r(k)=\left.\sum_{k=1}^{n}\left|\tilde{s}[k]^{2}\right| \bar{P}_{s}\left(u^{(k-1)}\right)\right|^{2} e^{j u}+\sum_{k=1}^{n} z[k]
$$

is complex Gaussian distributed with the joint conditional probability density function (pdf) of its real and imaginary parts, given $\tilde{s}[k]$ and $u^{(k-1)}, k=1,2, \cdots, n$, denoted as $\widetilde{\mathbf{s}}$ and $\mathbf{u}$, i.e.,

$$
f_{R^{(n)} \mid \tilde{\mathbf{s}}, \mathbf{u}}\left(R_{r}^{(n)}, R_{i}^{(n)} \mid \tilde{\mathbf{s}}, \mathbf{u}\right)=\frac{1}{2 \pi \sigma_{R^{(n)}}^{2}} \exp \left(-\frac{\left(R_{r}^{(n)}-\operatorname{Re}\left\{m_{R^{(n)}}\right\}\right)^{2}+\left(R_{i}^{(n)}-\operatorname{Im}\left\{m_{R^{(n)}}\right\}\right)^{2}}{2 \sigma_{R^{(n)}}^{2}}\right)
$$

where $m_{R^{(n)}}=\sum_{k=1}^{n}|\tilde{s}[k]|^{2}\left|\bar{P}_{s}\left(u^{(k-1)}\right)\right|^{2} e^{j u}$ is the conditional mean, and $\sigma_{R^{(n)}}^{2}=\sum_{k=1}^{n}|\tilde{s}[k]|^{2}\left|\bar{P}_{s}\left(u^{(k-1)}\right)\right|^{2} \sigma_{z}^{2}$ is the conditional variance of its real or imaginary part. The conditional pdf of $u^{(n)}$ is thus $f_{u^{(n)} \mid \tilde{\mathbf{s}}, \mathbf{u}}\left(u^{(n)} \mid \widetilde{\mathbf{s}}, \mathbf{u}\right)=f_{0}\left(u^{(n)}, \gamma_{\tilde{\mathbf{s}}, \mathbf{u}}^{(n)}\right)[20]$ where

$$
f_{0}(x, \gamma)=\frac{1}{2 \pi} e^{-\gamma}\left[1+\sqrt{4 \pi \gamma} \cos x e^{\gamma \cos ^{2} x} Q(-\sqrt{2 \gamma} \cos x)\right]
$$

$\gamma_{\tilde{\mathbf{s}}, \mathbf{u}}^{(n)}=\frac{\left|m_{R^{(n)}}\right|^{2}}{2 \sigma_{R^{(n)}}^{2}}=\frac{\left.\sum_{k=1}^{n}\left|\tilde{s}[k]^{2}\right| \bar{P}_{s}\left(u^{(k-1)}\right)\right|^{2}}{2 \sigma_{z}^{2}}$ is the conditional SNR of $R^{(n)}$, and $Q(x)=\frac{1}{\sqrt{2 \pi}} \int_{x}^{\infty} e^{-\frac{t^{2}}{2}} d t$ is the Q-function. Finally, the MSE for the estimation of $u$ at time index $n$ is 


$$
\sigma_{u^{(n)}}^{2}=\int_{-\pi}^{\pi}\left(u^{(n)}\right)^{2} f_{u^{(n)}}\left(u^{(n)}\right) d u^{(n)}
$$

where $f_{u^{(n)}}\left(u^{(n)}\right)=E_{\widetilde{\mathbf{s}}, \mathbf{u}}\left\{f_{u^{(n)} \mid \tilde{\mathbf{s}}, \mathbf{u}}\left(u^{(n)} \mid \widetilde{\mathbf{s}}, \mathbf{u}\right)\right\}$ is the unconditional pdf of $u^{(n)}$.

The exact evaluation of $\sigma_{u^{(n)}}^{2}$ is practically infeasible since the unconditional pdf of $u^{(n)}$ involves the expectation over the previously estimated $u^{(k-1)}$, which in turn requires the knowledge of the unconditional pdf of $u^{(k-1)}, k=1,2, \cdots, n$. Even the widely used modified Cramer-Rao bound (MCRB) analysis [21] is still infeasible since the calculation

$$
\operatorname{MCRB}\left(\sigma_{u^{(n)}}^{2}\right)=\frac{1}{E_{\widetilde{\mathbf{s}}, \mathbf{u}}\left\{E_{R^{(n)} \mid \tilde{\mathbf{s}}, \mathbf{u}}\left\{-\frac{\partial^{2} \ln f_{R^{(n)} \mid \tilde{\mathbf{s}}, \mathbf{u}}\left(R_{r}^{(n)}, R_{i}^{(n)} \mid \widetilde{\mathbf{s}}, \mathbf{u}\right)}{\partial u^{2}}\right\}\right\}}
$$

also requires that the unconditional pdf of $u^{(k-1)}, k=1,2, \cdots, n$, is known.

To avoid the direct evaluation of the MSE but still obtain a meaningful indication of it, a lower MSE bound of $\sigma_{u^{(n)}}^{2}$, referred to as Recursive MSE Bound, is obtained as (see Appendix)

$$
\operatorname{MSEB}\left(\sigma_{u^{(n)}}^{2}\right)=\frac{\sqrt{\bar{\gamma}^{(n)} \pi^{2}+1}}{\pi\left(\bar{\gamma}^{(n)}\right)^{\frac{3}{2}}} \sinh ^{-1}\left(\sqrt{\bar{\gamma}^{(n)}} \pi\right)-\frac{1}{\bar{\gamma}^{(n)}}
$$

where $\bar{\gamma}^{(n)}$ is the average SNR of $R^{(n)}$, which is recursively determined by

$$
\bar{\gamma}^{(n)}= \begin{cases}\frac{1}{2 N} \gamma, & n=1 \\ \bar{\gamma}^{(n-1)}+\frac{1}{2} \gamma \int_{-\pi}^{\pi}\left|\bar{P}_{s}\left(u^{(n-1)}\right)\right|^{2} \frac{\sqrt{\bar{\gamma}^{(n-1)} \pi^{2}+1}}{2 \pi\left(\bar{\gamma}^{(n-1)}\left(u^{(n-1)}\right)^{2}+1\right)^{3 / 2}} d u^{(n-1)}, & n>1\end{cases}
$$

and $\sinh ^{-1}(x)=\ln \left(x+\sqrt{x^{2}+1}\right)$ denotes the inverse hyperbolic sine function.

Eq. (29) also applies to DBS after the beam scanning scheme is incorporated. For a linear hybrid array of two side-by-side subarrays with $N$ elements, we have $\bar{P}_{s}(u)=\frac{\sin \left(N \frac{u}{2}\right)}{N \sin \left(\frac{u}{2}\right)}$ and the incremental of $\bar{\gamma}^{(n)}$ in (30) will be $\frac{1}{2} \gamma \int_{-\pi}^{\pi}\left|\bar{P}_{s}\left(\frac{u^{(n-1)}}{N}\right)\right|^{2} \frac{\sqrt{\bar{\gamma}^{(n-1)} \pi^{2}+1}}{2 \pi\left(\bar{\gamma}^{(n-1)}\left(u^{(n-1)}\right)^{2}+1\right)^{3 / 2}} d u^{(n-1)}$. Since the 
average SNR is only updated every $N$ samples (assuming a one sample subframe), the convergence rate is reduced by a factor of $N$.

\section{Simulation Results}

The proposed AoA estimation algorithms are simulated using a planar hybrid array of four interleaved subarrays and a planar hybrid array of four side-by-side subarrays respectively, as shown in Fig. 1. Each subarray has 16 elements arranged as a 4 by 4 matrix and there are 2 subarrays placed along $\mathrm{x}$-axis and $\mathrm{y}$-axis respectively, resulting in a large array of 64 elements. The element spacing is half of the wavelength, i.e., $d=\frac{\lambda_{c}}{2}$. The incident angles of the received signal are set to 40 degree in zenith and 0 degree in azimuth, corresponding to $u_{x}=2.0194$ and $u_{y}=0$ respectively. The SNR per antenna element is set to $-10 \mathrm{~dB}$. The reference signal is a realization of a complex Gaussian distributed random signal.

The simulation results using DBT with updating coefficient $\mu=0.001$ for the hybrid array of interleaved subarrays are shown in Fig. 4 and Fig. 5. Fig. 4 shows the estimated phases of the cross-correlations along $\mathrm{x}$-axis and $\mathrm{y}$-axis versus the number of iterations respectively. The estimated values after 400 iterations are 1.979 and 0.016 respectively, corresponding to 39.05 degree in zenith and 0.46 degree in azimuth. Fig. 5 shows the normalized array factors for an interleaved subarray and the overall hybrid array respectively. We see that the grating lobe in the subarray is suppressed after combining the four subarrays. The final beamwidth of the hybrid array is similar to that of an interleaved subarray as the aperture of the interleaved subarray is similar to that of the hybrid array.

The simulation results using DBS with updating coefficient $\mu=0.001$ and $\beta=0.25$ for the hybrid array of side-by-side subarrays are shown in Fig. 6 to Fig. 9. In this case, the correct $\arg \left\{R_{x}\right\}$ and $\arg \left\{R_{y}\right\}$ values are 1.7943 and 0 respectively. The possible $u_{x}$ values are -2.6930 , $-1.1222,0.4486,2.0194$, and 3.5902, and the possible $u_{y}$ values are $-3.1416,-1.5708,0,1.5708$, and 3.1416. There are total 25 different beams to scan, so that there are 25 subframes in one 
scanning frame. The number of samples in one subframe is set to 4 in the simulation and thus one scanning frame has 100 signal samples. Fig. 6 depicts the estimated $\arg \left\{R_{x}\right\}$ and $\arg \left\{R_{y}\right\}$ versus the number of iterations respectively for 4 scanning frames. Compared with Fig. 4 , we see that the convergence is slower than that of DBT using interleaved subarrays. Fig. 7 shows the $u_{x}$ and $u_{y}$ values when the beams are sequentially scanned. Fig. 8 shows the estimated signal powers for all beams during the beam scanning. We see that the peak power in each scanning frame appears when the beam with the forth value of $u_{x}$ and the third value of $u_{y}$ is scanned, and thus this beam is detected as the correct one. The estimated $u_{x}$ and $u_{y}$ values after 4 scanning frames are 1.970 and -0.022 respectively which correspond to 38.84 degree in zenith and -0.64 degree in azimuth. Finally, Fig. 9 shows the normalized array factors for the side-by-side subarray and the overall hybrid array respectively. We see that after combining the subarrays the beamwidth of the hybrid array is reduced significantly. Compared with Fig. 5, we also see that, though the subarray patterns are different for the interleaved and the side-by-side subarrays, the overall hybrid arrays after the AOA estimation are very similar. This means that the subarray configuration does not affect the overall beamforming accuracy though the beamforming algorithms have different complexities and convergence speeds.

Finally, the simulated MSEs for the DBT algorithm under different SNRs per subarray are plotted in Fig. 10, which are obtained after averaging over 100 independent simulations. Note that the subarray SNR of $\gamma=5 \mathrm{~dB}$ corresponds to the $-10 \mathrm{~dB}$ element SNR, since the subarray has 16 elements, and two subarray correlations are combined for the AoA estimation (thus the gain is $10 \log (16) \mathrm{dB}+3 \mathrm{~dB}=15 \mathrm{~dB})$. The calculated recursive MSE bounds are also displayed on the same figure. We see that at lower SNR the MSE bound is rather loose. However as SNR increases, the recursive MSE bound becomes tighter.

\section{CONCLUSIONS}

A hybrid adaptive antenna array with two different subarray configurations and the associated AoA estimation and beamforming algorithms are proposed to simplify the 
implementation of large adaptive antenna arrays, especially at mm-wave frequencies. It has been shown that the proposed DBT and DBS algorithms can estimate the AoA information for hybrid arrays of interleaved and side-by-side subarrays respectively without the knowledge of a reference signal or signal synchronization. The DBT algorithm has lower complexity and converges faster than the DBS algorithm but it requires the employment of interleaved subarrays, whereas the DBS algorithm can be used for beam acquisition in a more practical hybrid array of side-by-side subarrays. The proposed AoA estimation can be formulated as a phase estimation problem under recursive nuisance parameters and a recursive MSE bound is derived to give a meaningful indication of the estimation performance. The effects of mutual coupling and other practical impairments in the hybrid array will be studied in our future research.

\section{APPENDIX: RECURSIVE MSE BOUND}

The lower MSE bound is obtained by replacing $f_{u^{(n)}}\left(u^{(n)}\right)$ with a known phase distribution determined only by the average SNR of $R^{(n)}$, defined by

$$
\bar{\gamma}^{(n)}=E_{\tilde{\mathbf{s}} \mathbf{u}}\left\{\gamma_{\tilde{\mathbf{s}}, \mathbf{u}}^{(n)}\right\}=\frac{1}{2} \sum_{k=1}^{n} E\left\{\frac{|\tilde{s}[k]|^{2}}{\sigma_{z}^{2}}\right\} E_{u^{(k-1)}}\left\{\bar{P}_{s}\left(u^{(k-1)}\right)^{2}\right\}=\frac{1}{2} \gamma \sum_{k=1}^{n} E_{u^{(k-1)}}\left\{\left.\bar{P}_{s}\left(u^{(k-1)}\right)\right|^{2}\right\} .
$$

The known pdf of $u^{(n)}$ is chosen as the phase distribution under Rayleigh fading channel [20] since $\mid \tilde{s}[n]$ in $(21)$ is Rayleigh distributed, i.e, $f_{u^{(n)}}\left(u^{(n)}\right)=f_{1}\left(u^{(n)}, \bar{\gamma}^{(n)}\right)$ where

$$
f_{1}(x, \gamma)=\frac{1}{2 \pi\left(1+\gamma \sin ^{2} x\right)}+\frac{\sqrt{\gamma} \cos x}{2 \pi\left(1+\gamma \sin ^{2} x\right)^{3 / 2}}\left(\frac{\pi}{2}+\tan ^{-1} \frac{\sqrt{\gamma} \cos x}{\sqrt{1+\gamma \sin ^{2} x}}\right) .
$$

The justification is that if the subarray radiation pattern is omni-directional, i.e., $\bar{P}_{s}(u)=1$, $f_{1}\left(u^{(n)}, \bar{\gamma}^{(n)}\right)$ will be the true phase distribution in Rayleigh fading channel.

Since $\left|\bar{P}_{s}(u)\right| \leq 1$ in practice, which will lead to a reduced SNR, the actual MSE in (27) will be always greater than that calculated using $f_{1}\left(u^{(n)}, \bar{\gamma}^{(n)}\right)$, i.e,

$$
\sigma_{u^{(n)}}^{2} \geq \int_{-\pi}^{\pi}\left(u^{(n)}\right)^{2} f_{1}\left(u^{(n)}, \bar{\gamma}^{(n)}\right) d u^{(n)}
$$


Therefore, the right-hand side of (33) represents a lower MSE bound, denoted as $\operatorname{MSEB}\left(\sigma_{u^{(n)}}^{2}\right)$.

Furthermore, from (31) $\bar{\gamma}^{(1)}$ can be determined as

$$
\bar{\gamma}^{(1)}=\frac{1}{2} \gamma E_{u^{(0)}}\left\{\left.\bar{P}_{s}\left(u^{(0)}\right)\right|^{2}\right\}=\frac{1}{4 \pi} \gamma \int_{-\pi}^{\pi}\left|\frac{\sin \left(N u^{(0)}\right)}{N \sin \left(u^{(0)}\right)}\right|^{2} d u^{(0)}=\frac{1}{2 N} \gamma
$$

where the initial $u^{(0)}$ is assumed to be uniformly distributed in $[-\pi, \pi)$, and $\bar{\gamma}^{(n)}$ for $n>1$ can be recursively determined as

$$
\begin{aligned}
\bar{\gamma}^{(n)} & =\frac{1}{2 N} \gamma+\frac{1}{2} \gamma \int_{-\pi}^{\pi}\left|\bar{P}_{s}\left(u^{(1)}\right)\right|^{2} f_{1}\left(u^{(1)}, \bar{\gamma}^{(1)}\right) d u^{(1)}+\cdots+\frac{1}{2} \gamma \int_{-\pi}^{\pi}\left|\bar{P}_{s}\left(u^{(n-1)}\right)\right|^{2} f_{1}\left(u^{(n-1)}, \bar{\gamma}^{(n-1)}\right) d u^{(n-1)} \\
& =\bar{\gamma}^{(n-1)}+\frac{1}{2} \gamma \int_{-\pi}^{\pi}\left|\bar{P}_{s}\left(u^{(n-1)}\right)\right|^{2} f_{1}\left(u^{(n-1)}, \bar{\gamma}^{(n-1)}\right) d u^{(n-1)}, \text { for } n>1 .
\end{aligned}
$$

The MSE bound calculated based on the above recursively determined average SNR is thus called Recursive MSE Bound.

At high SNR $f_{0}(x, \gamma)$ in (26) can be approximated as the Gaussian distribution

$$
f_{0}(x, \gamma) \approx \sqrt{\frac{\gamma}{\pi}} \exp \left(-\gamma x^{2}\right), \quad \text { for }-\pi \leq x<\pi
$$

and accordingly $f_{1}(x, \gamma)$ can be approximated as

$$
f_{1}(x, \gamma) \approx \frac{\sqrt{\gamma \pi^{2}+1}}{2 \pi\left(2 x^{2}+1\right)^{3 / 2}}, \quad \text { for }-\pi \leq x<\pi,
$$

after normalizing it to satisfy the condition $\int_{-\pi}^{\pi} f_{1}(x, \gamma) d x=1$. A closed-form equation of the MSE bound is then obtained as

$$
\begin{aligned}
\operatorname{MSEB}\left(\sigma_{u^{(n)}}^{2}\right) & =\int_{-\pi}^{\pi}\left(u^{(n)}\right)^{2} f_{1}\left(u^{(n)}, \bar{\gamma}^{(n)}\right) d u^{(n)} \\
& \approx \int_{-\pi}^{\pi}\left(u^{(n)}\right)^{2} \frac{\sqrt{\bar{\gamma}^{(n)} \pi^{2}+1}}{2 \pi\left(\bar{\gamma}^{(n)}\left(u^{(n)}\right)^{2}+1\right)^{3 / 2}} d u^{(n)} \\
& =\frac{\sqrt{\bar{\gamma}^{(n)} \pi^{2}+1}}{\pi\left(\bar{\gamma}^{(n)}\right)^{\frac{3}{2}}} \sinh ^{-1}\left(\sqrt{\bar{\gamma}^{(n)}} \pi\right)-\frac{1}{\bar{\gamma}^{(n)}} .
\end{aligned}
$$




\section{REFERENCES}

[1] Y. Jay Guo, Advances in Mobile Radio Access Networks, Artech House, Inc., 2004.

[2] Robert J. Mailloux, Phased Array Antenna Handbook, Artech House, Inc. 2005.

[3] D. Rogstad, A. Mileant, and T. Pham, Antenna Arraying Techniques in the Deep Space Network, Wiley-IEEE, 2003.

[4] H. Meinel, "Commercial applications of millimeterwaves history, present status, and future trends." IEEE Transactions on Microwave Theory and Techniques, Vol. 43, No. 7, July 1995, pp. 1639-1653.

[5] J. I. Agbinya, et al, Advances in Broadband Communication and Networks, River Publishers, 2008.

[6] H. Zirath, T. Masuda, R. Kozhuharov and M. Ferndahl, "Development of 60-GHz front-end circuits for a highdata-rate communication system,” IEEE Journal of Solid-State Circuits, Vol. 39, No. 10, October 2004, pp. 1640-1649.

[7] C. H. Doan, S. Emami, D. A. Sobel, A. M. Niknejad, and R. W. Brodersen, "Design considerations for $60 \mathrm{GHz}$ CMOS radios,” IEEE Communications Magazine, Vol. 42, No. 12, December 2004, pp. 132-140.

[8] T. Do-Hong and P. Russer, "Signal processing for wideband smart antenna array applications," IEEE Microwave Magazine, March 2004, pp. 57-67.

[9] Y. Jay Guo, J. Bunton, V. Dyadyuk and X. Huang, "Hybrid adaptive antenna array," Australian provisional patent, TW8245/AU, February 2009.

[10] A. P. Goffer, M Kam, and P. R. Herczfeld, "Design of phased arrays in terms of random subarrays," IEEE Transactions on Antennas and Propagation, Vol. 42, No. 6, June 1994, pp. 820-826.

[11] R. L. Haupt, "Optimized weighting of uniform subarrays of unequal sizes," IEEE Transactions on Antennas and Propagation, Vol. 55, No. 4, April 2007, pp.1207-1210.

[12] R. J. Mailloux, "Subarray technology for large scanning arrays," The Second European Conference on Antennas and Propagation (EuCAP2007), 11-16 November 2007.

[13] H. Krim and M. Viberg, "Two decades of array signal processing research," IEEE Signal Processing Magazine, July 1996, pp. 67-94.

[14] T. Xia, Y. Zheng, Q. Wan, and X. Wang, "Decoupled estimation of 2-D angles of arrival using two parallel uniform linear arrays," IEEE Transactions on Antennas and Propagation, Vol. 55, No. 9, September 2007, pp. $2627-2632$.

[15] P. Stoica and A. Nehorai, "MUSIC, maximum likelihood, and Cramer-Rao bound," IEEE Transactions on Acoustics, Speech, and Signal Processing, Vol. 37, No. 5, May 1989, pp. 720-741.

[16] M. Viberg and B. Ottersten, "Sensor array processing based on subspace fitting," IEEE Transactions on Signal Processing, Vol.39, No.5, May 1991, pp. 1110-1121. 
[17] B. Ottersten, M. Viberg, P. Stoica, and A. Nehorai, "Exact and large sample maximum likelihood techniques for parameter estimation and detection in array processing," in Radar Array Processing, S. Haykin, J. Litva, and T.J. Shepherd, Ed., pp. 99-151, Springer-Verlag, 1993.

[18] P. Stoica and K. C. Sharman, "Maximum likelihood methods for direction-of-arrival estimation," IEEE Transactions on Acoustics, Speech, and Signal Processing, Vol.38, No.7, July 1990, pp. 1132-1143.

[19] A. Papoulis and S. U. Pillai, Probability, Random Variables and Stochastic Processes, Fourth Edition, McGraw-Hill, 2002.

[20] R. Reggiannini, "A fundamental lower bound to the performance of phase estimators over Rician-fading channels," IEEE Transactions on Communications, Vol. 45, No. 7, July 1997, pp. 775-778.

[21] A. N. D'Andrea, U. Mengali, and R. Reggiannini, "The modified Cramer-Rao bound and its application to synchronization problems," IEEE Transactions on Communications, Vol. 42, No. 2/3/4, February/March/April 1994, pp. 1391-1399.

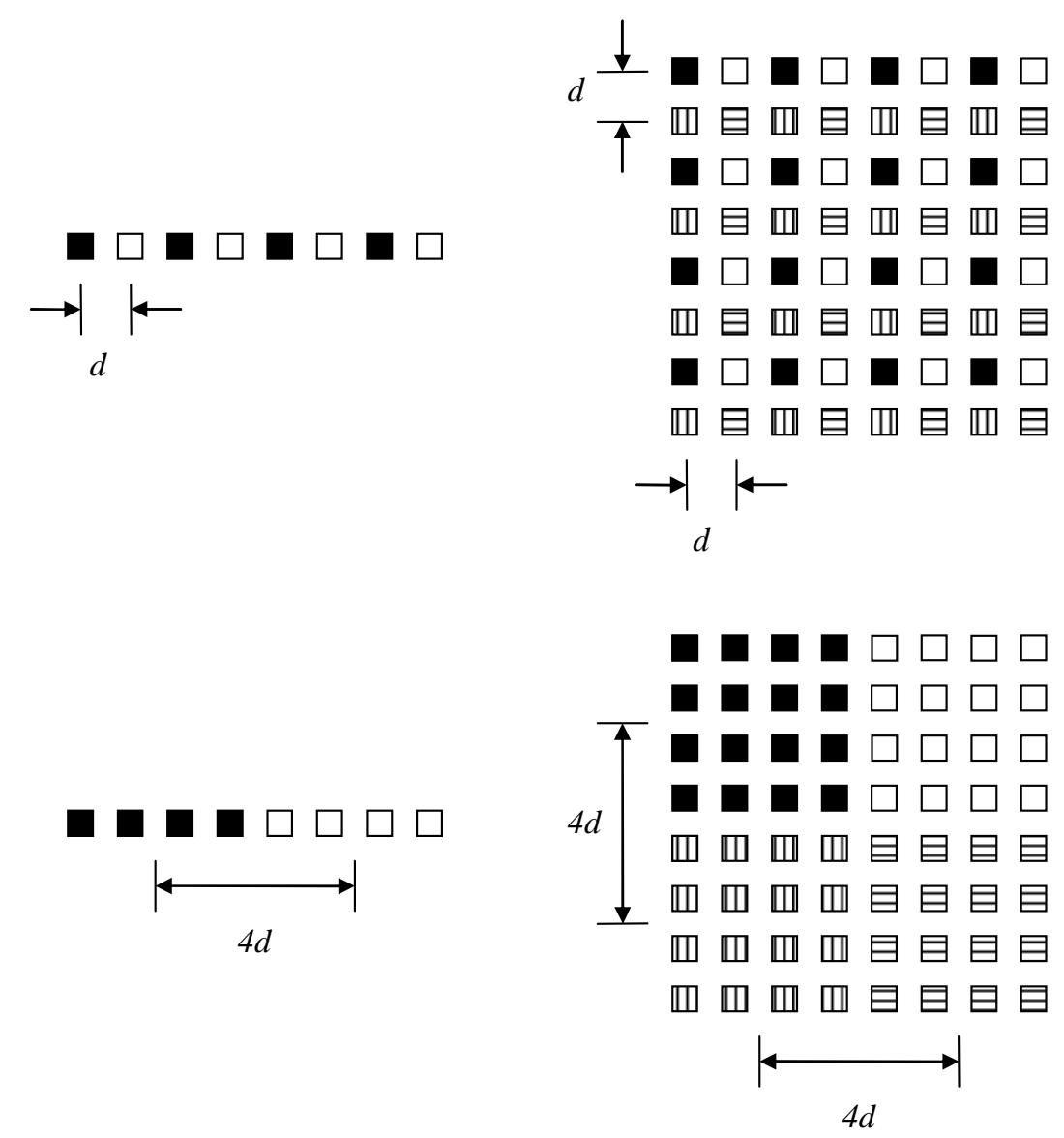

Fig. 1. Hybrid antenna arrays of interleaved linear subarrays (top left) and planar subarrays (top right) versus hybrid antenna arrays of side-by-side linear subarrays (bottom left) and planar subarrays (bottom right). Different subarrays are shown in different fill patterns. 


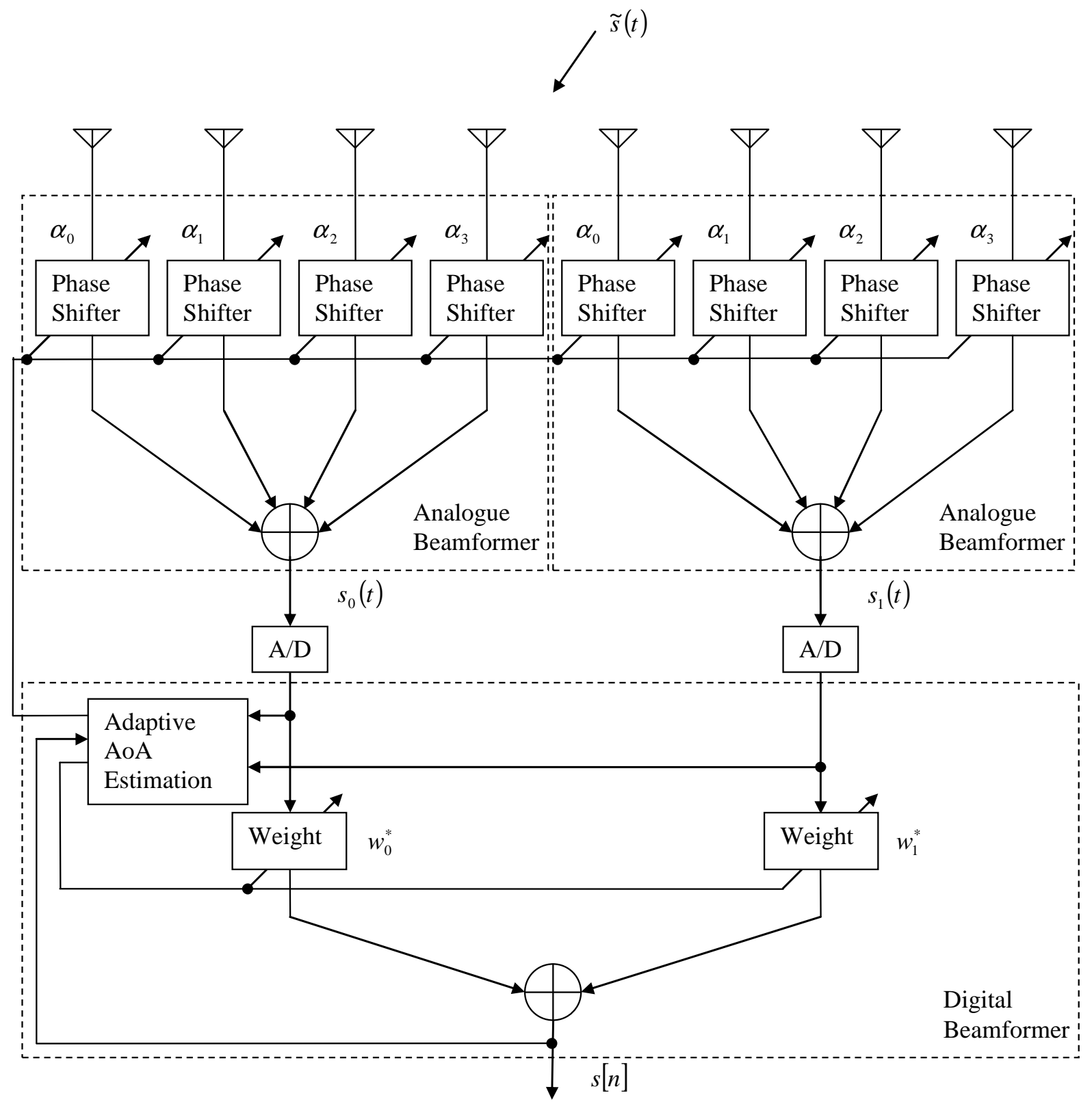

Fig. 2. Illustration of hybrid beamformer structure with two side-by-side linear subarrays. 


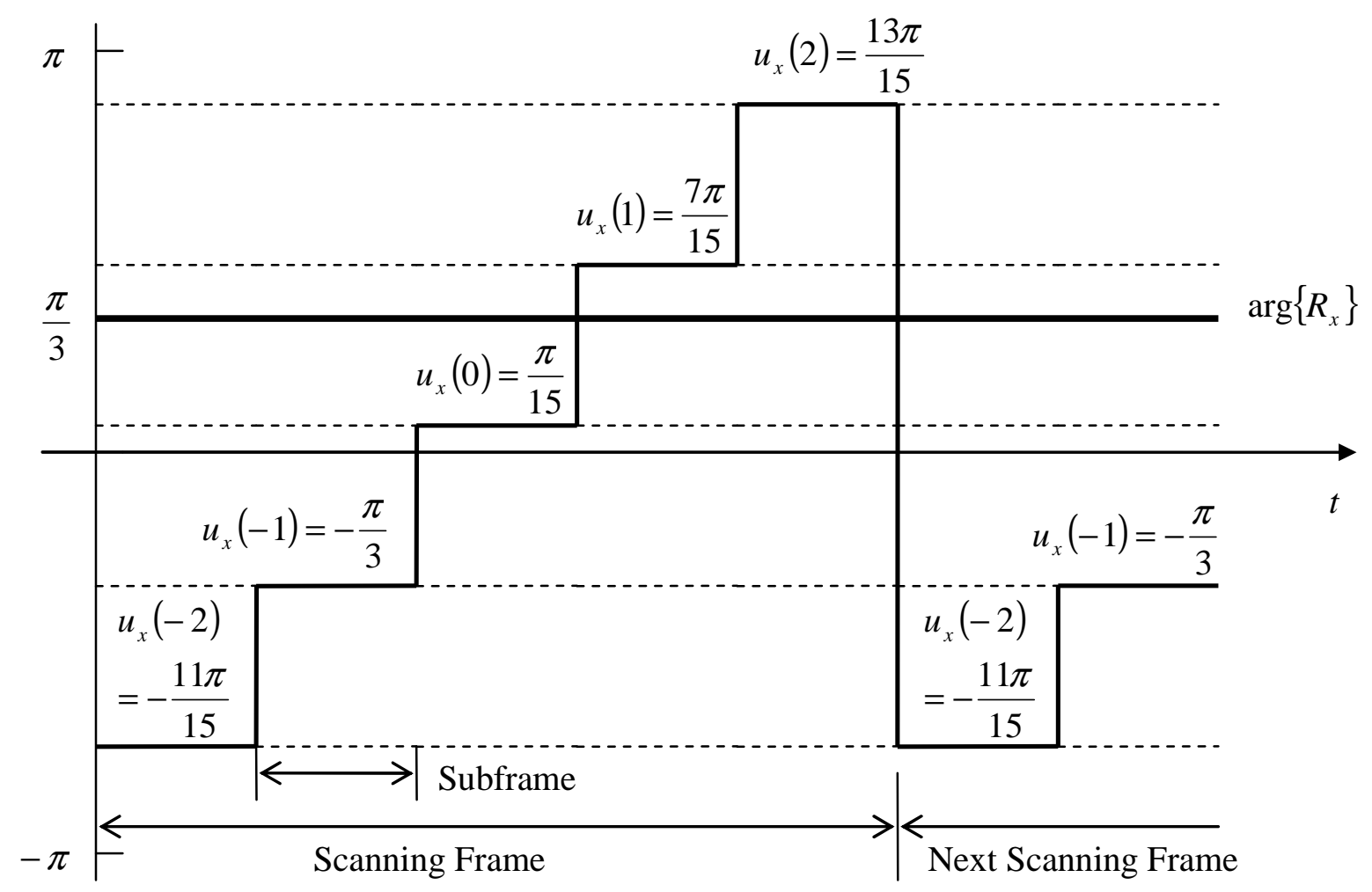

Fig. 3. Illustration of scanning frame and subframes for beam search.

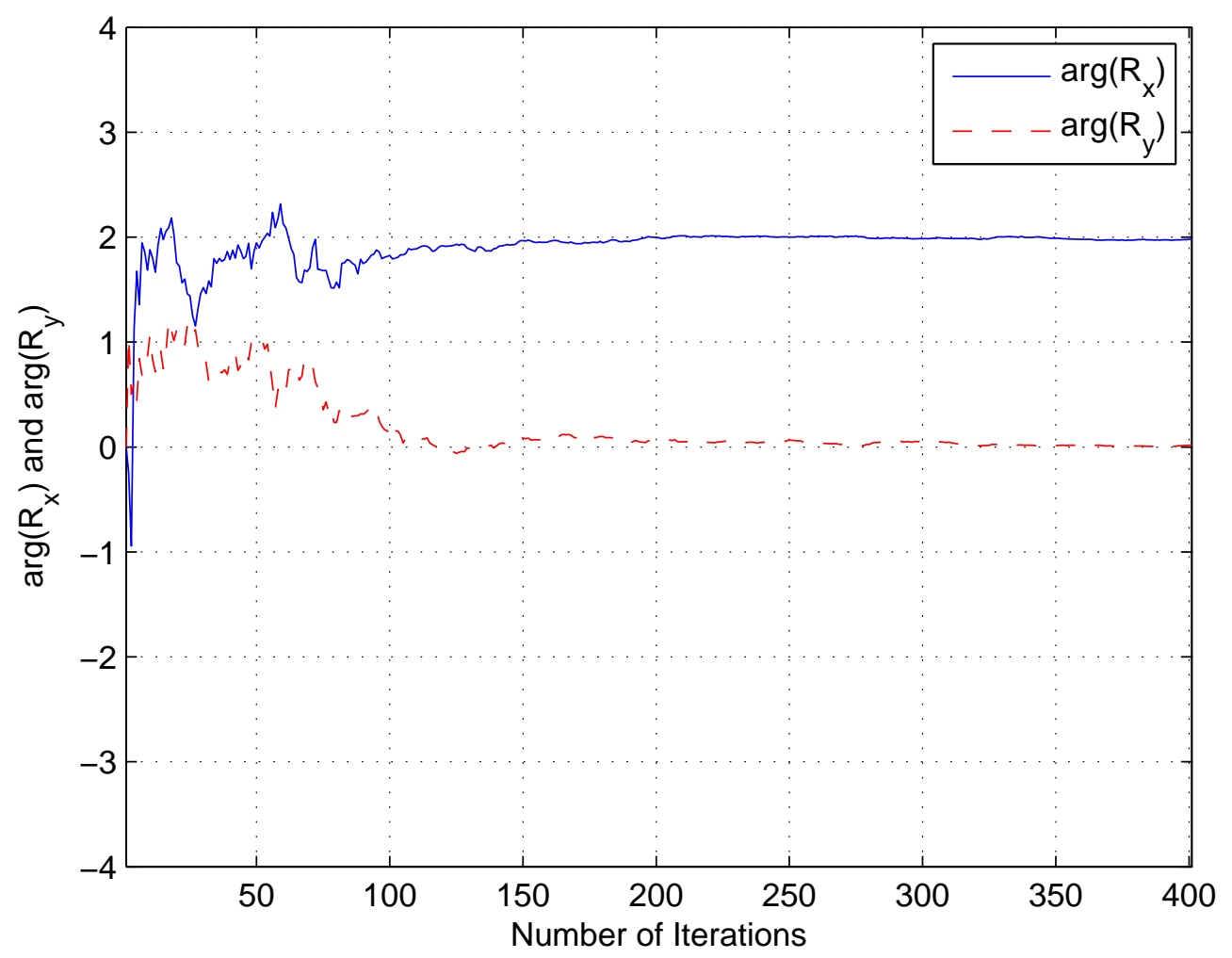

Fig. 4. Estimated phases of cross-correlations versus the number of iterations using DBT for hybrid array of interleaved subarrays. 


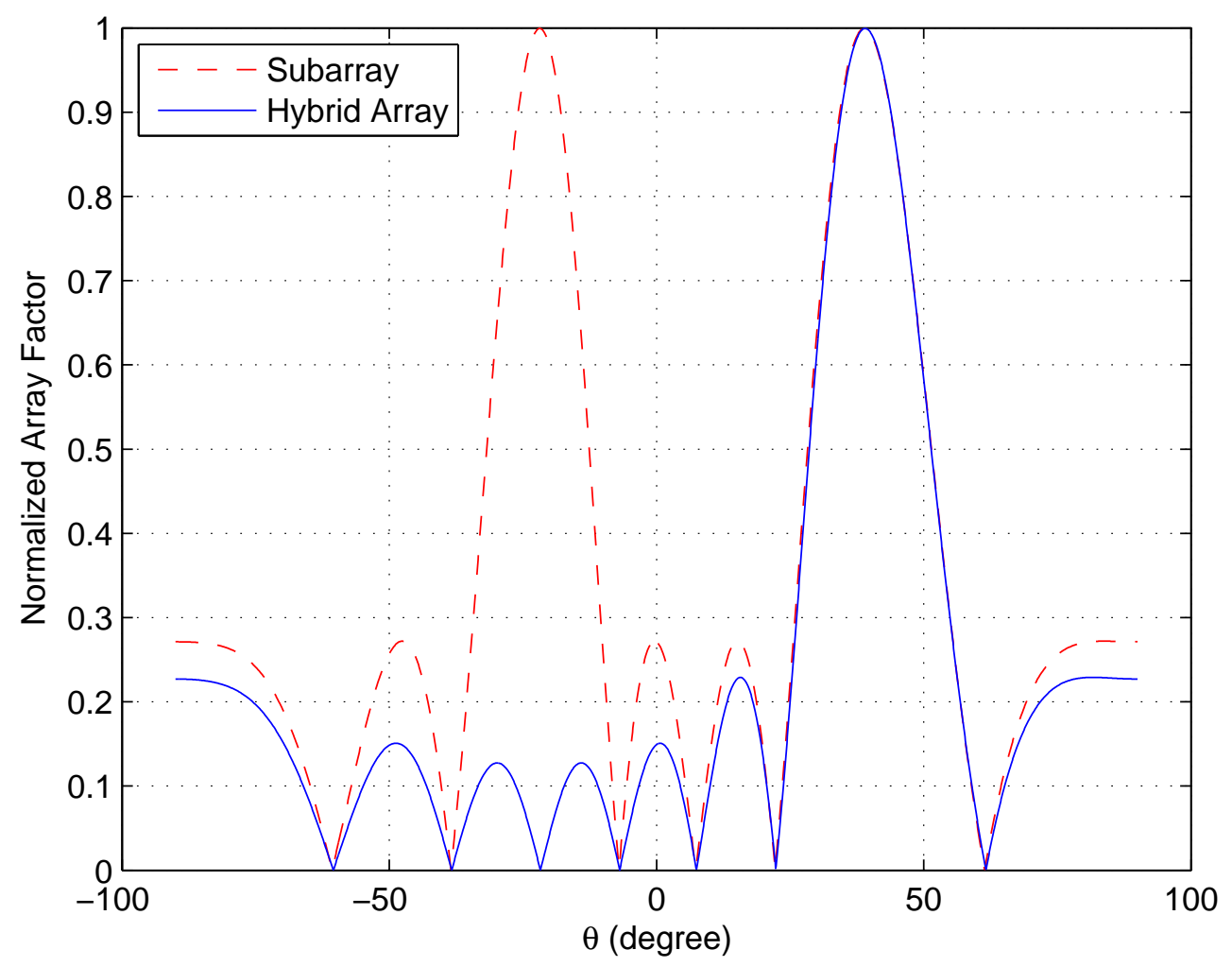

Fig. 5. Normalized array factors of an interleaved subarray and hybrid array $(\phi=0)$.

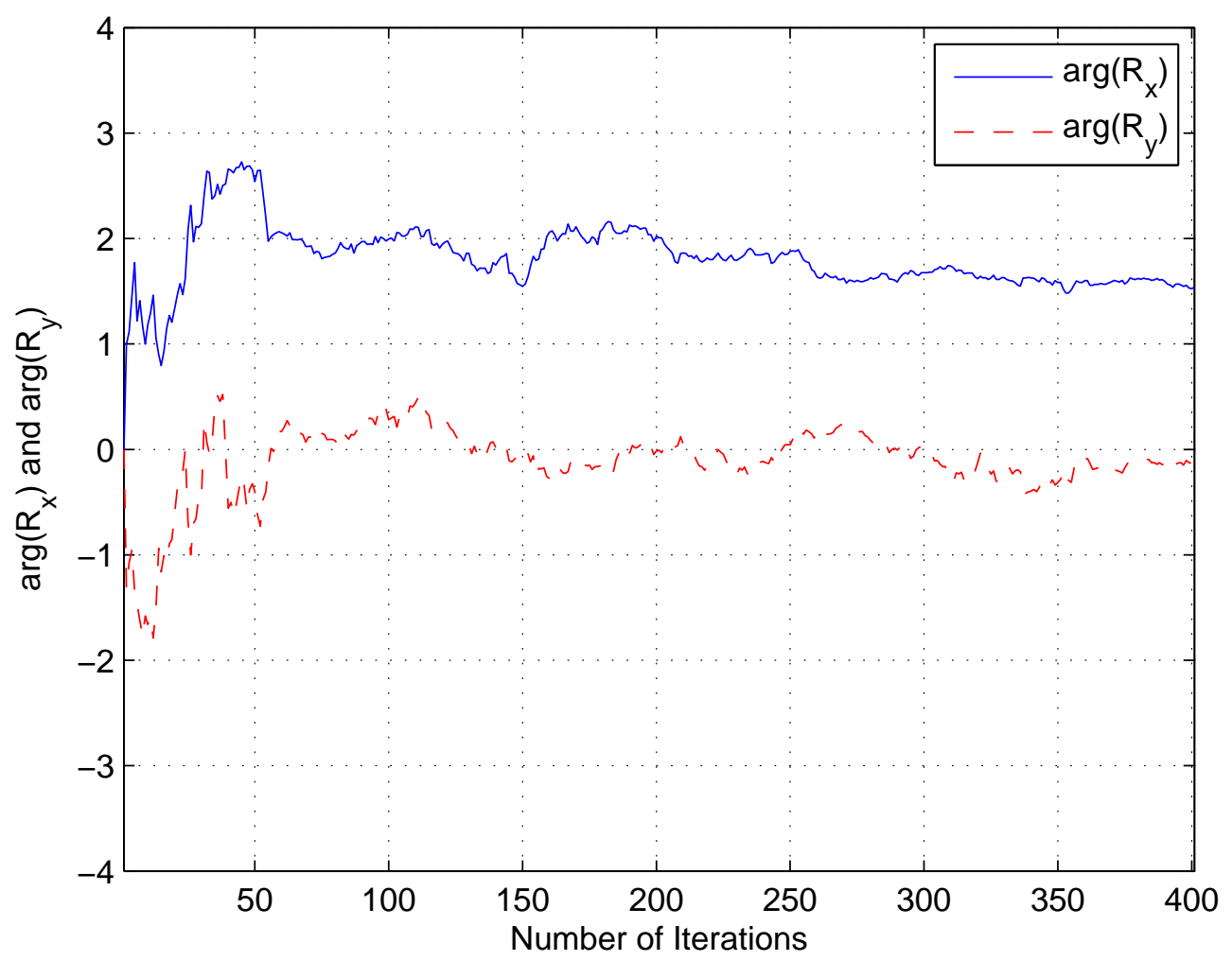

Fig. 6. Estimated phases of cross-correlations versus the number of iterations using DBS for hybrid array of side-by-side subarrays. 


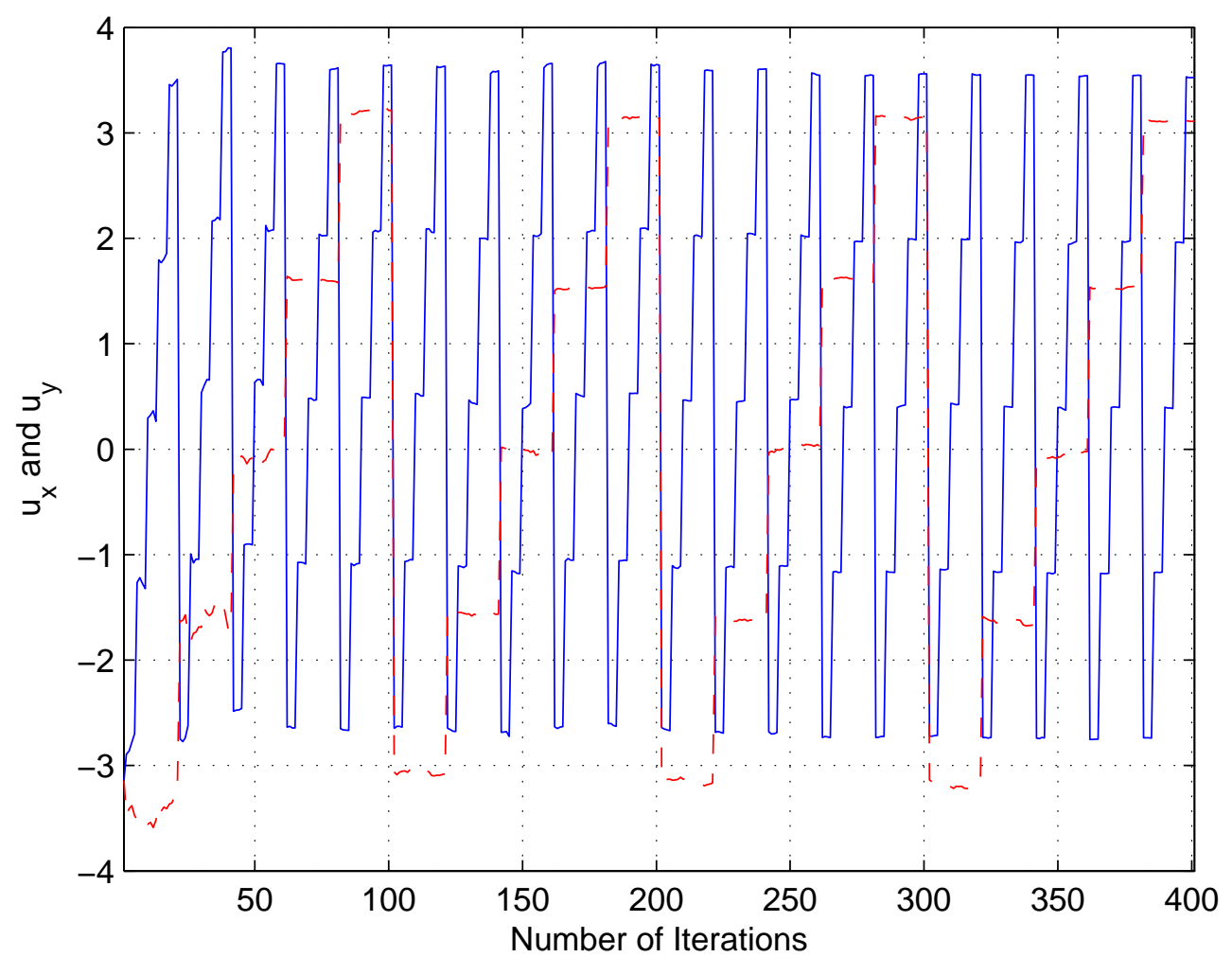

Fig. 7. Beam search via $u_{x}$ (solid line) and $u_{y}$ (dashed line) scanning.

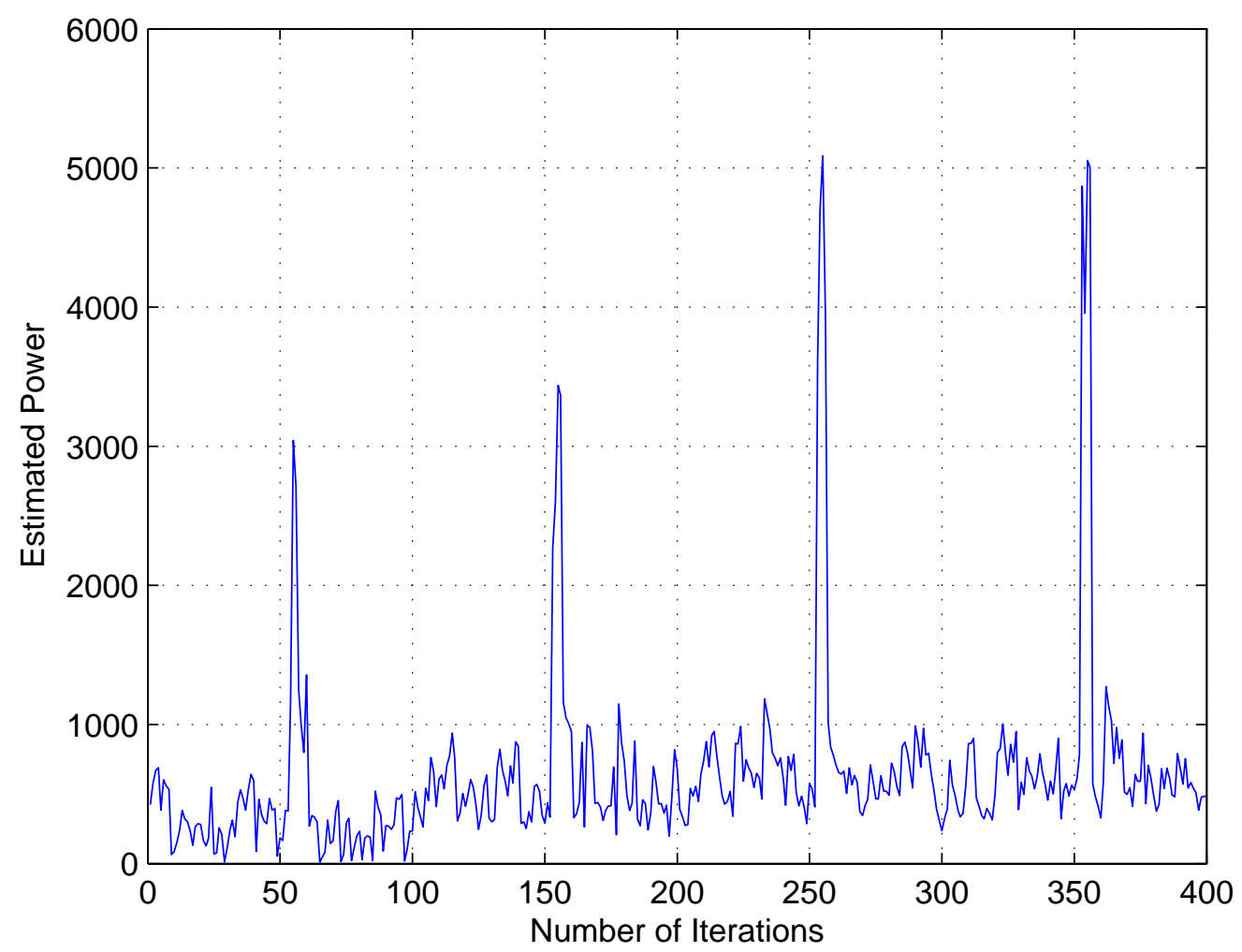

Fig. 8. Power profile of different beams. Peak power indicates the correct beam. 


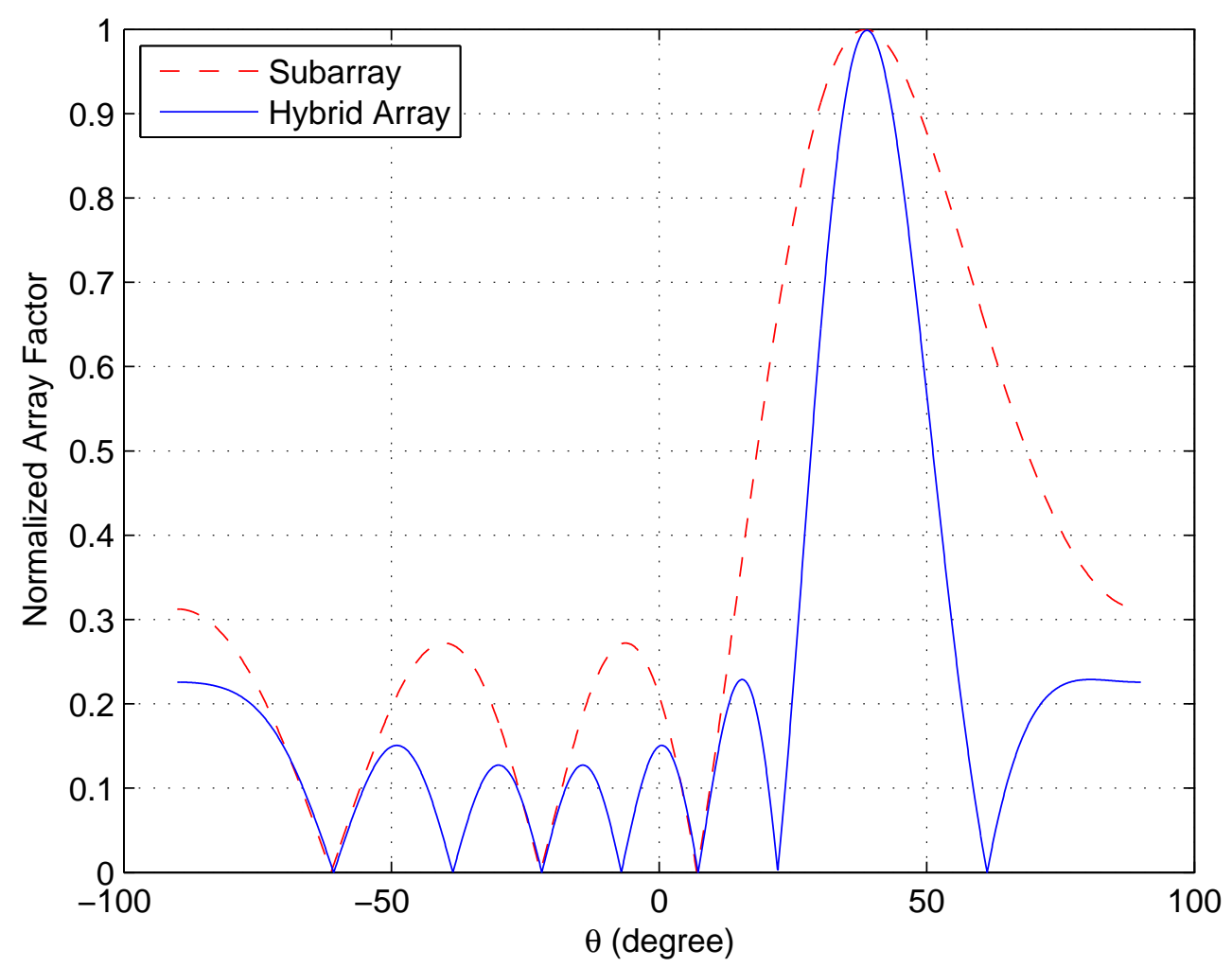

Fig. 9. Normalized array factors of a side-by-side subarray and hybrid array $(\phi=0)$.

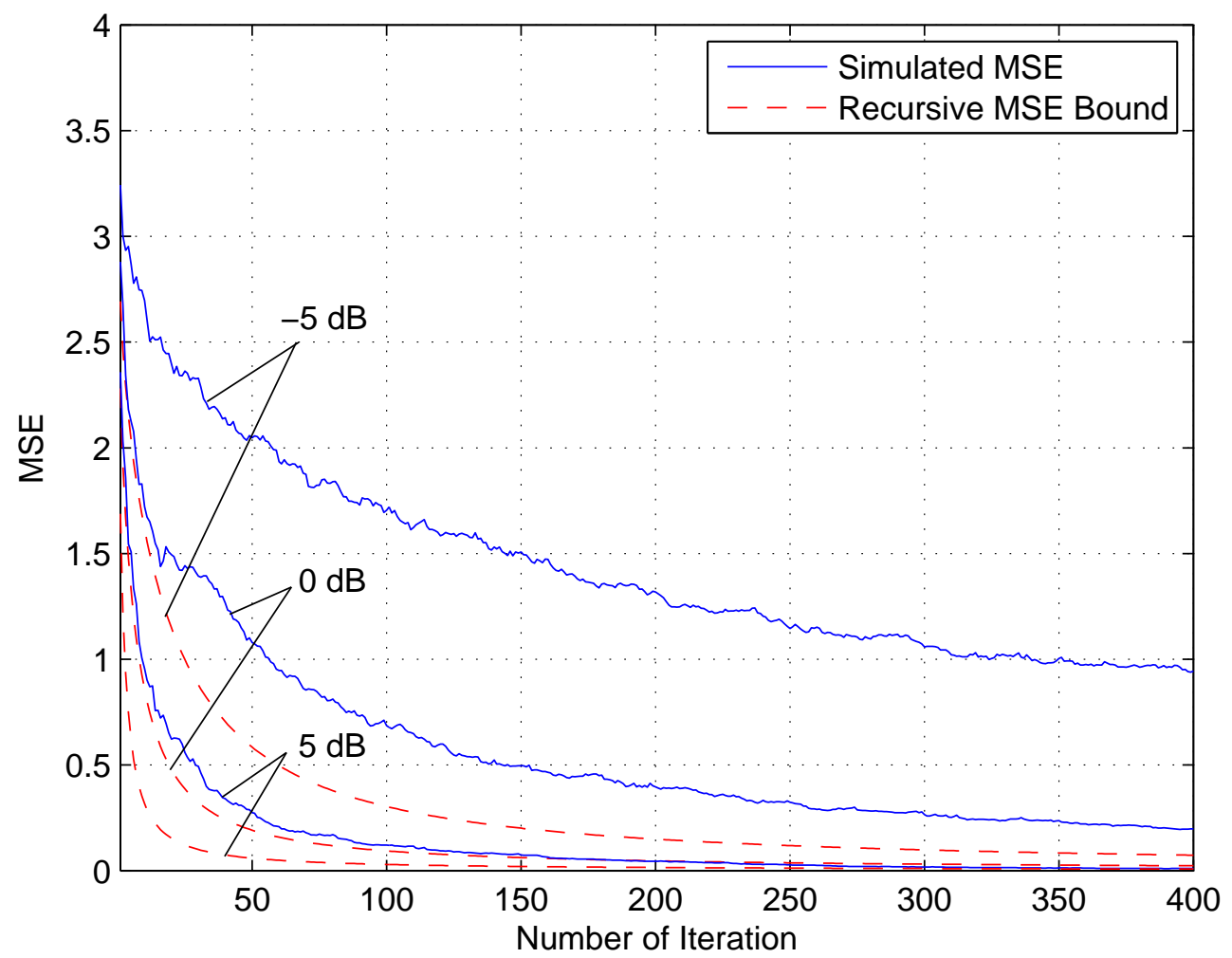

Fig. 10. Simulated MSEs and recursive MSE bounds under -5, 0, and $5 \mathrm{~dB}$ subarray SNRs. 